\title{
Coupled-bunch instability for arbitrary multibunch configurations
}

\author{
Gabriele Bassi@, ${ }^{*}$ Alexei Blednykh๑, ${ }^{\dagger}$ and Victor Smaluk $\oplus^{\ddagger}$ \\ Brookhaven National Laboratory, Upton, New York 11973-5000, USA
}

(Received 22 March 2021; accepted 6 December 2021; published 25 January 2022)

\begin{abstract}
The coupled-bunch instability for arbitrary multibunch configurations is investigated in its generality. The theoretical framework adopted is based on the general eigenvalue analysis based on the known formulas of the complex frequency shifts for the uniform multibunch configuration case. Closed formulas are derived for special cases. For the configuration consisting of a uniform filling pattern with a gap of missing bunches, the theoretical results are found to be in good agreement with measurements of the transverse coupled-bunch instability driven by the resistive wall impedance performed at the NSLS-II storage ring.
\end{abstract}

DOI: 10.1103/PhysRevAccelBeams.25.014402

\section{INTRODUCTION}

The design of modern light sources, with their goal to store beams with low-emittance and high-average current, requires detailed studies to minimize detrimental effects on the beam quality induced by short- and long-range wakefields. These wakefields are generated by the electromagnetic interaction of the circulating beam with the storage ring vacuum components and, acting back on the beam, determine current dependent instability thresholds that can pose severe limitations to the achievable beam current [1]. An example of a long-range wakefield potentially driving a coupled-bunch instability is represented by the resistive wall wakefield studied in this paper. Light sources operate with a variety of multibunch configurations that differ from the uniform one. A common mode of operation, which is adopted by the NSLS-II storage ring [2], consists of a bunch train with a gap in the uniform filling pattern [3], aimed to combat multibunch instabilities driven by ions. The gap, however, introduces transient beam loading, an effect that has a negative impact on the performance of the rf-system, especially when higher-harmonic cavities for bunch lengthening are used [4]. Several light sources have therefore considered configurations with multigaps to improve performance. Modes of operation with hybrid filling patterns aimed to accommodate special experimental conditions such as in time resolved experiments [4-8] are

\footnotetext{
Corresponding author. gbassi@bnl.gov

†blednykh@bnl.gov

†vsmalyuk@bnl.gov
}

Published by the American Physical Society under the terms of the Creative Commons Attribution 4.0 International license. Further distribution of this work must maintain attribution to the author(s) and the published article's title, journal citation, and DOI. also commonly used. The analysis of the stability of nonuniform multibunch configurations is therefore an important issue.

The coupled-bunch instability theory for uniform filling patterns is well developed $[9,10]$. Attempts for a general theoretical analysis in the case of arbitrary filling patterns have been discussed, with various degrees of approximation, in Refs. [11-15]. In this paper, we adopt the general theoretical framework developed in Ref. [16], where starting from a system of coupled Vlasov equations governing the evolution of the phase space densities of the circulating bunches, it is shown that the stability analysis is reduced to the formulation of an eigenvalue problem defined by the complex frequency shifts of the uniform filling pattern case. The solution of the eigenvalue problem allows the determination of the eigenvalue with the largest imaginary part, thus characterizing the growth rate of the fastest coupled bunch instability, with the instability threshold obtained by equating the fastest growth rate to the radiation damping rate. The analysis is restricted to the dipole mode of oscillation and to the case of zero chromaticity. We therefore neglect shortrange wakefields and the important head-tail damping effect at positive chromaticity, as well as the effect from a transverse bunch-by-bunch feedback system, all crucial ingredients for stabilizing standard modes of operation in storage rings. Such effects are taken into account, for example, in the nested head-tail Vlasov solver discussed in Ref. [15], and can be studied numerically with particle tracking codes such as the Vlasov-Fokker-Planck solver space [16]. Our choice to neglect the aforementioned effects is motivated by our intent to simplify the comparison between theory and measurements, by isolating the coupled-bunch interaction as the dominant effect determining the instability threshold.

The paper is organized as follows. In Sec. II, we discuss the resistive wall impedance budget of the NSLS-II storage ring, as the major contributor to the transverse coupled bunch instability. In Sec. III, we revisit the theory of the 
coupled-bunch instability for arbitrary multibunch configurations developed in Ref. [16], and derive closed formulas for some specific cases. In Sec. IV, we discuss measurements of the vertical coupled-bunch instability performed at the NSLS-II storage ring and compare them with the results of the eigenanalysis presented in Sec. III.

\section{RESISTIVE WALL IMPEDANCE}

In NSLS-II, the major contribution to the transverse coupled-bunch instability is given by the resistive wall impedance, $Z_{1}^{\perp}$, of the storage ring components, assuming the standard thick wall approximation. Its use is justified by the smallness of the coating thickness of the vacuum components and by the fact that the lowest frequency contribution to the coupled-bunch instability threshold is given by $\operatorname{Re} Z_{1}^{\perp}$ sampled at $(1-q) \omega_{0}$, where $\omega_{0}$ is the angular revolution frequency and $q$ the fractional part of the betatron tune $\nu_{\beta}=\omega_{\beta} / \omega_{0}$, where $\omega_{\beta}$ is the betatron frequency [17]. For one component of the storage ring, the thick wall formula, valid for $|\omega| \leq c / s_{0}$, reads [18]

$$
\begin{aligned}
Z_{1}^{\perp}(\omega) & =(1-i \operatorname{sgn}(\omega)) \frac{Z_{0} s_{0} L \sqrt{s_{0} c|\omega|}}{2 \pi \omega b^{4}}, \\
s_{0} & =\sqrt[3]{\frac{2 b^{2}}{Z_{0} \sigma_{c}}}
\end{aligned}
$$

where $Z_{0}$ is the impedance of free space and $c$ the speed of light in vacuum, and $L, \sigma_{\mathrm{c}}$, and $b$ are the length, electrical conductivity, and half aperture of the impedance structure, respectively. The electrical conductivity $\sigma_{c}$ of the material of the storage ring vacuum components is shown in Table I, together with the relevant contributions to the vertical resistive wall impedance budget, where a distinction is made between contributions from arcs and straight sections. In Table I, the different elements have the following meaning: vacuum chamber $(\mathrm{CH})$, fast corrector (FC), and damping wiggler (DW). The contribution from $N$ elements is weighted by the average of the beta function over the length of the impedance structure

$$
\bar{\beta}_{y, i}=\frac{1}{L_{i}} \int_{s_{i}-L_{i} / 2}^{s_{i}+L_{i} / 2} d s^{\prime} \beta_{y}\left(s^{\prime}\right), \quad i=1, \ldots, N
$$

where $s_{i}$ is the location of the center of the element along the ring. Eliminating $s_{0}$ in Eq. (1), the total average impedance $\bar{Z}_{1}{ }^{\perp}$ is defined as

$$
\bar{Z}_{1}^{\perp}(\omega)=(1-i \operatorname{sgn}(\omega)) \frac{\sqrt{2 Z_{0} c|\omega|}}{2 \pi \omega} K, \quad K=\sum_{i} \alpha_{i},
$$

where $\alpha_{i}=f_{i} L_{i} \bar{\beta}_{y, i} /\left(\sqrt{\sigma_{c, i}} b_{i}^{3}\right)$ and $f_{i}$ is a geometric factor: $f_{i}=1$ for components with circular geometry, $f_{i}=\pi^{2} / 12$ for components with parallel geometry. In Table I, the value

TABLE I. NSLS-II resistive wall impedance.

\begin{tabular}{lcc}
\hline \hline Material & Symbol & $\sigma_{c}(\mathrm{MS} / \mathrm{m})$ \\
\hline \multirow{3}{*}{ Stainless steel } & Electrical conductivity of resistive wall components & \\
Aluminum & $\mathrm{SS}$ & 1.35 \\
Inconel & $\mathrm{Al}$ & 31.6 \\
& $\mathrm{Inc}$ & 0.775 \\
\hline \hline
\end{tabular}

\begin{tabular}{lccccc}
\hline \hline \multicolumn{7}{c}{ Main contributions from arcs } \\
\hline Element & Material & $L(\mathrm{~m})$ & $\bar{\beta}_{y}(\mathrm{~m})$ & $b(\mathrm{~mm})$ & $\alpha / K(\%)$ \\
\hline $\mathrm{CH} 1$ & $\mathrm{Al}$ & 536.4 & 17.6 & 12.5 & 70.8 \\
$\mathrm{CH} 2$ & $\mathrm{SS}$ & 11.5 & 8 & 12.5 & 3.3 \\
\hline \hline
\end{tabular}

\begin{tabular}{lcccccc}
\hline \hline \multicolumn{7}{c}{ Main contributions from straight sections } \\
\hline Cell & Element & Material & $L(\mathrm{~m})$ & $\bar{\beta}_{y}(\mathrm{~m})$ & $b(\mathrm{~mm})$ & $\alpha / K(\%)$ \\
\hline 1 & CH & Al & 5.2 & 22 & 12.5 & $6.2^{\mathrm{a}}$ \\
1 & FC & Inc & 0.37 & 8.3 & 12.5 & $4.4^{\mathrm{b}}$ \\
6 & CH & Al & 5.2 & 22 & 12.5 & $3.4^{\mathrm{c}}$ \\
6 & FC & Inc & 0.37 & 8.7 & 12.5 & $4.7^{\mathrm{d}}$ \\
8 & DW & Al & 7.5 & 4 & 5.75 & $7.2^{\mathrm{e}}$ \\
\hline \hline
\end{tabular}

${ }^{\mathrm{a}}$ Including the contribution from cells 9, 13, 15, 25, 27, and 29, which are identical to cell 1 .

${ }^{\mathrm{b}}$ Total contribution from all odd cells, which have two FCs each.

${ }^{\mathrm{c}}$ Including the contribution from cells 14, 20, and 26, which are identical to cell 6 .

${ }^{\mathrm{d}}$ Total contribution from all even cells, which have two FCs each.

${ }^{\mathrm{e}}$ Including the contribution from cells 18 and 28 , which are identical to cell 8 . 

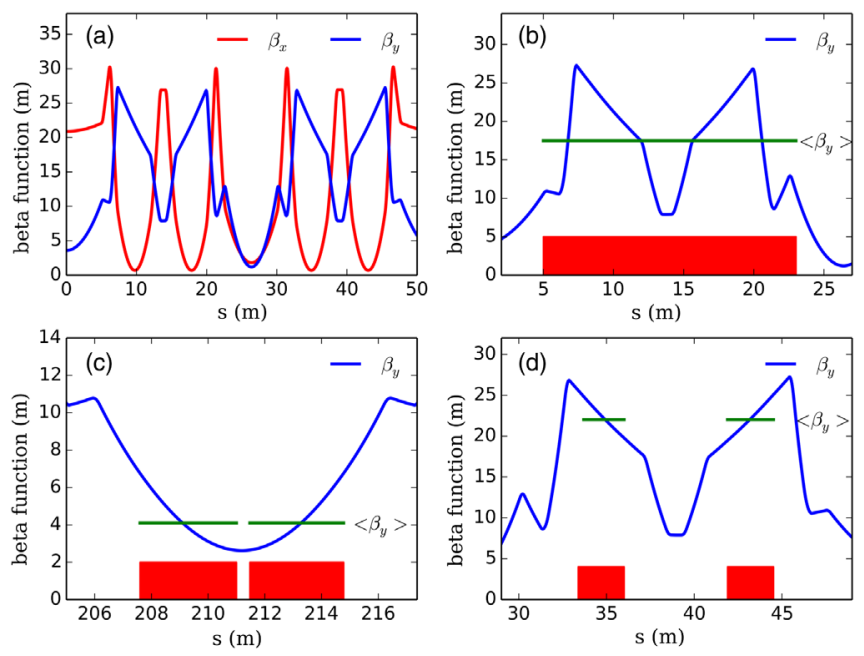

FIG. 1. (a) One superperiod of the NSLS-II DBA lattice consisting of two cells with short and long straight sections. Vertical beta function (magenta trace) variation and its average (green trace) over the length of the impedance element (red rectangle): (b) aluminum vacuum chamber in the first half superperiod; (c) DWs in cell 8; (d) bending magnet chamber in cell 1 .

$\alpha / K$ refers to the contribution in percentage of the various impedance elements. Figure 1(a) shows the beta functions of two cells of the NSLS-II storage ring [2], which adopts a double bend achromat lattice with 30 periods, thus dividing the storage ring in 30 cells. The two cells shown in Fig. 1(a) correspond to one super-period of the lattice. The main contribution to the total impedance budget, approximately $70 \%$, is given by the aluminum vacuum chamber, as shown in Table I. Its contribution from one half of the lattice superperiod is shown in Fig. 1(b). Figures 1(c) and 1(d) show contributions from storage ring components localized in straight sections, with the contributions weighted by the average beta function over the impedance structure length according to Eq. (2).

\section{EIGENVALUE ANALYSIS}

We adopt the theoretical framework of the coupled-bunch instability for arbitrary filling patterns developed in Ref. [16], which leads to the formulation of an eigenvalue problem defined in terms of the complex frequency shifts of the uniform filling pattern case. Here, we briefly revisit the theory, more details can be found in Ref. [16]. The most general multibunch configuration is defined by $h$ bunches, where $h$ is the harmonic number (representing the number of available rf-buckets), with $N_{m}$ particles $(m=0, \ldots, h-1)$, circulating in the storage ring with the reference particles of the bunches separated by the distance $d=C / h$, where $C$ is the ring circumference. The starting framework of the theoretical formulation is defined by a system of $h$-coupled Vlasov equations, which give the time evolution of the phase space densities $\Psi_{m}$ associated with the $m$ th bunch. The phase space coordinates are $(\tau, \delta, x, p)$, where $\tau$ is arrival time, $\delta=$ $\left(E-E_{0}\right) / E_{0}$ the relative energy deviation, where $E_{0}$ is the energy of the reference particle in electron volts, and $x$ and $p_{x}$ are the transverse position and transverse momentum, respectively. Here $x$ represents the transverse coordinate, either horizontal or vertical. We assume linearized longitudinal equations of motion and neglect quantum radiation effects. For the evolution of the dipole moment, the last assumption has the effect to neglect an exponential decay determined by the radiation damping constant. The transverse phase space densities $\Psi_{m}\left(\tau, \delta, x, p_{x}, t\right)$, associated to the $m$ th bunch with $N_{m}$ particles, satisfy the following system of $h$-coupled Vlasov equations for $0 \leq m \leq h-1$

$$
\begin{gathered}
\frac{\partial \Psi_{m}}{\partial t}-\eta \delta \frac{\partial \Psi_{m}}{\partial \tau}+\frac{\omega_{s}^{2}}{\eta} \tau \frac{\partial \Psi_{m}}{\partial \delta}+p_{x} \frac{\partial \Psi_{m}}{\partial x}-\omega_{\beta}^{2} x \frac{\partial \Psi_{m}}{\partial p_{x}} \\
-A_{x} \sum_{k=0}^{+\infty} \sum_{m^{\prime}=0}^{h-1} N_{m^{\prime}}\left[\int_{-\infty}^{\tau} d \tau^{\prime} \bar{W}_{1}\left(\tau-\tau^{\prime}+a_{m^{\prime} m}^{k} T_{0}\right)\right. \\
\left.\times \int_{-\infty}^{+\infty} d x^{\prime} x^{\prime} \rho_{m^{\prime}}\left(\tau^{\prime}, x^{\prime}, t-a_{m^{\prime} m}^{k} T_{0}\right)\right] \frac{\partial \Psi_{m}}{\partial p_{x}}=0,
\end{gathered}
$$

where $\rho_{m}(\tau, x, t)=\int d \delta \int d p_{x} \Psi_{m}\left(\tau, \delta, x, p_{x}, t\right)$ and $a_{m^{\prime} m}^{k}=$ $k+\left(m^{\prime}-m\right) / h, A_{x}=e \omega_{\beta} /\left(T_{0} E_{0}\right)$ [19], where $T_{0}$ is the revolution period, $e$ is the electron charge, $\omega_{\beta}$ is the betatron frequency, $\eta=\alpha-1 / \gamma^{2}$ is the slippage factor where $\alpha$ is the momentum compaction factor, $\omega_{s}$ is the synchrotron frequency, $\bar{W}_{1}$ is the total average transverse wake function, and $\sum_{m=0}^{h-1} N_{m}=N h=N_{T}$ is the total number of particles in the filling pattern, with $N$ the number of particles per bunch in the uniform filling pattern case. We neglect longitudinal wake fields, so for the longitudinal equations of motion we consider $\dot{\tau}=-\eta \delta, \quad \dot{\delta}=\omega_{s}^{2} \tau / \eta, \equiv d / d t$. The equation for the evolution of the dipole moments $\left\langle x_{m}\right\rangle=$ $\int d \tau d \delta d x d p_{x} x \Psi_{m}$ and $\left\langle p_{x_{m}}\right\rangle=\int d \tau d \delta d x d p_{x} p_{x} \Psi_{m}$ can be found by integrating by parts the Vlasov equations using the boundary conditions for $\Psi_{m}$. Using the approximation $\rho_{m}(\tau, x, t)=\lambda(\tau) \mu_{m}(x, t)$, where $\mu_{m}(\tau, t)=$ $\int d \tau \rho_{m}(\tau, x, t)$ is the transverse distribution density of the $m$ th bunch and it is assumed that all bunches have the same stationary longitudinal distribution density $\lambda(\tau)$, it can be shown [16] that $\left\langle x_{m}\right\rangle$ satisfies

$$
\begin{aligned}
\left\langle\ddot{x}_{m}\right\rangle+\omega_{\beta}^{2}\left\langle x_{m}\right\rangle= & -A_{x} \sum_{k=0}^{+\infty} \sum_{m^{\prime}=0}^{h-1} f\left(a_{m^{\prime} m}^{k} T_{0}\right) \\
& \times N_{m^{\prime}}\left\langle x_{m^{\prime}}\right\rangle\left(t-a_{m^{\prime} m}^{k} T_{0}\right),
\end{aligned}
$$

where $f(x) \equiv \int d \tau \int d \tau^{\prime} \bar{W}_{1}\left(\tau-\tau^{\prime}+x\right) \lambda(\tau) \lambda\left(\tau^{\prime}\right)$. In deriving Eq. (5), it is assumed that the transverse wake function $\bar{W}_{1}(\tau)$ varies slowly over the support of the longitudinal distribution density $\lambda(\tau)$. Proceeding by omitting the brackets in the equations, i.e., $x_{m}$ should be understood as $\left\langle x_{m}\right\rangle$, and defining the coupled-bunch mode $\tilde{x}_{\mu}$ by 


$$
\begin{gathered}
\tilde{x}_{\mu}(t)=\sum_{m=0}^{h-1} x_{m}(t) e^{-i 2 \pi m \mu / h} \text { and } \\
x_{m}(t)=\frac{1}{h} \sum_{\mu=0}^{h-1} \tilde{x}_{\mu}(t) e^{i 2 \pi m \mu / h},
\end{gathered}
$$

from Eq. (5) it follows the modes $\tilde{x}_{\mu}$ are coupled and satisfy the equations of motion

$$
\begin{aligned}
\ddot{\tilde{x}}_{\mu}(t)+\omega_{\beta}^{2} \tilde{x}_{\mu}(t)= & -\frac{A_{x}}{h} \sum_{k=0}^{\infty} f\left(k \frac{T_{0}}{h}\right) e^{i 2 \pi \mu k / h} \\
& \times \sum_{\mu^{\prime}=0}^{h-1} \tilde{x}_{\mu^{\prime}}\left(t-k \frac{T_{0}}{h}\right) \sum_{m=0}^{h-1} N_{m} e^{i 2 \pi m\left(\mu^{\prime}-\mu\right) / h} .
\end{aligned}
$$

In the uniform filling pattern case, $N_{m}=N$, thus from the orthogonality condition $\sum_{m=0}^{h-1} e^{i 2 \pi m\left(\mu^{\prime}-\mu\right) / h}=h \delta_{\mu^{\prime} \mu}$, where $\delta_{\mu^{\prime} \mu}$ is the Kronecker delta, it follows that the modes $\tilde{x}_{\mu}$ are uncoupled and satisfy

$$
\ddot{\tilde{x}}_{\mu}(t)+\omega_{\beta}^{2} \tilde{x}_{\mu}(t)=-A_{x} N \sum_{k=0}^{\infty} f\left(k \frac{T_{0}}{h}\right) e^{i 2 \pi \mu k / h} .
$$

Since $x_{m} \in \mathbb{R}$, it follows that $\tilde{x}_{\mu}=\tilde{x}_{h-\mu}^{\star}$, thus it is natural to define the multibunch mode by

$$
\begin{aligned}
x_{m}^{(\mu)}(t) & =\frac{1}{h}\left(\tilde{x}_{\mu}(t) e^{i 2 \pi m \mu / h}+\tilde{x}_{h-\mu}(t) e^{-i 2 \pi m \mu / h}\right) \\
& =\frac{2}{h}\left(\operatorname{Re} \tilde{x}_{\mu}(t) \cos \frac{2 \pi \mu m}{h}-\operatorname{Im} \tilde{x}_{\mu}(t) \sin \frac{2 \pi \mu m}{h}\right) .
\end{aligned}
$$

We assume a perturbative solution of Eq. (8) of the form

$$
\tilde{x}_{\mu}(t)=a_{\mu} e^{-i\left(\omega_{\beta}+\Omega\right) t}, \quad|\Omega| \ll \omega_{\beta}, \quad \Omega \in \mathbb{C} .
$$

Defining $\quad \tau^{-1} \equiv \operatorname{Im} \Omega, \quad \omega_{r} \equiv \operatorname{Re} \Omega, \quad$ and $\quad$ assuming $\operatorname{Im} \tilde{x}_{\mu}(0)=0$, it follows that the multibunch mode $x_{m}^{(\mu)}$ takes the form

$$
x_{m}^{(\mu)}(t)=a e^{\frac{t}{\tau}} \cos \left[\frac{2 \pi \mu m}{h}-\left(\omega_{\beta}+\omega_{r}\right) t\right],
$$

where $a=2 \operatorname{Re} \tilde{x}_{\mu}(0) / h$. By inserting Eq. (11) in (8) we obtain the eigenvalue equation determining the transverse stability of the multibunch configuration given by Eq. (46) of Ref. [16]

$$
\begin{aligned}
(\mathbf{B}-\Omega \mathbf{I}) \mathbf{a} & =0, \\
B_{\mu \mu^{\prime}} & =\frac{\Omega_{\mu}^{U}}{N_{T}} \sum_{m=0}^{h-1} N_{m} e^{i 2 \pi m\left(\mu^{\prime}-\mu\right) / h},
\end{aligned}
$$

where $\mathbf{a}=\left[a_{0}, \ldots, a_{h-1}\right]^{T}$ is the eigenvector corresponding to the eigenvalue $\Omega$, and $\Omega_{\mu}^{U}$ are the eigenvalues of the uniform filling pattern case $\left(B_{\mu \mu^{\prime}}=\Omega_{\mu}^{U}\right.$ if $\mu^{\prime}=\mu$, 0 otherwise), and are given by

$$
\Omega_{\mu}^{U}=-i \frac{I_{0}}{2 T_{0} E_{0}} \sum_{p=-\infty}^{+\infty}\left|\tilde{\lambda}\left(p^{\prime}\right)\right|^{2} \bar{Z}_{1}^{\perp}\left[p^{\prime}\right]
$$

where $p^{\prime}=(p h+\mu) \omega_{0}+\omega_{\beta}, \omega_{0}=2 \pi / T_{0}, I_{0}$ is the average current which can be equivalently expressed as $I_{0}=e \sum_{m=0}^{h-1} N_{m} / T_{0}=e N h / T_{0}=e N_{T} / T_{0}$, and $\bar{Z}_{1}^{\perp}$ is the total average transverse impedance related to the transverse wakefield $W_{1}$ by $Z_{1}^{\perp}(\omega)=i \int d \tau e^{i \omega \tau} W_{1}(\tau)$, $W_{1}(\tau)=-i /(2 \pi) \int d \omega e^{-i \omega \tau} Z_{1}^{\perp}(\omega) . \tilde{\lambda}(\omega)$ is the Fourier transform of the longitudinal distribution density, assumed to be given and the same for all bunches.

Solving for the characteristic polynomial $p(\Omega)=\mid \mathbf{B}-$ $\Omega \mathbf{I} \mid=0$ and assuming $h$ distinct eigenvalues $\Omega_{m}$, the general solution $\tilde{x}_{\mu}^{g}(t)$ is given by

$$
\tilde{x}_{\mu}^{g}(t)=\sum_{m=0}^{h-1} c_{m} a_{\mu m} e^{-i\left(\omega_{\beta}+\Omega_{m}\right) t},
$$

where $\mathbf{a}_{m}=\left[a_{0 m}, \ldots, a_{h-1 m}\right]^{T}$ are the eigenvectors associated to the eigenvalues $\Omega_{m}$.

The complex frequency shift $\Omega_{\mu}^{U}$ given by Eq. (14) is computed with parameters listed in Table II and $\bar{Z}_{1} \perp$ given by Eq. (3). The growth rate $\tau_{\mu}^{-1}=\operatorname{Im} \Omega_{\mu}^{U}$ and coherent tune shift $\nu_{r, \mu}=\omega_{r, \mu} / \omega_{0}$, where $\omega_{r, \mu}=\operatorname{Re} \Omega_{\mu}^{U}$, are shown in Figs. 2(a) and 2(b), respectively, with $I_{0}$ evaluated at the instability threshold $I_{\text {th }}$, which is determined by setting $\operatorname{Im} \Omega_{\mu}^{U}=1 / \tau_{y}$ and by finding the mode $\mu$ which maximizes $f(\mu) \equiv-\sum_{p=-\infty}^{+\infty}\left|\tilde{\lambda}\left(p^{\prime}\right)\right|^{2} \operatorname{Re} \bar{Z}_{1}^{\perp}\left[p^{\prime}\right]$, i.e.,

TABLE II. Parameters for the NSLS-II 3DWs lattice.

\begin{tabular}{lccc}
\hline \hline Parameter & Symbol & Value & Unit \\
\hline Energy & $E_{0}$ & 3 & $\mathrm{GeV}$ \\
Revolution period & $T_{0}$ & 2.64 & $\mu \mathrm{s}$ \\
Harmonic number & $\mathrm{h}$ & 1320 & \\
Momentum compaction & $\alpha$ & 0.00037 & \\
Synchrotron tune & $\nu_{s}$ & 0.007 & \\
Horizontal tune & $\nu_{x}$ & 32.22 & \\
Vertical tune & $\nu_{y}$ & 16.26 & \\
rf-Voltage & $V_{\mathrm{rf}}$ & 3 & $\mathrm{MV}$ \\
Transverse damping time & $\tau_{x, y}$ & 22.5 & $\mathrm{~ms}$ \\
Longitudinal damping time & $\tau_{s}$ & 11.9 & $\mathrm{~ms}$ \\
Energy spread & $\sigma_{\delta}$ & 0.00087 & \\
Bunch length & $\sigma_{t}$ & 14.5 & $\mathrm{ps}$ \\
\hline \hline
\end{tabular}



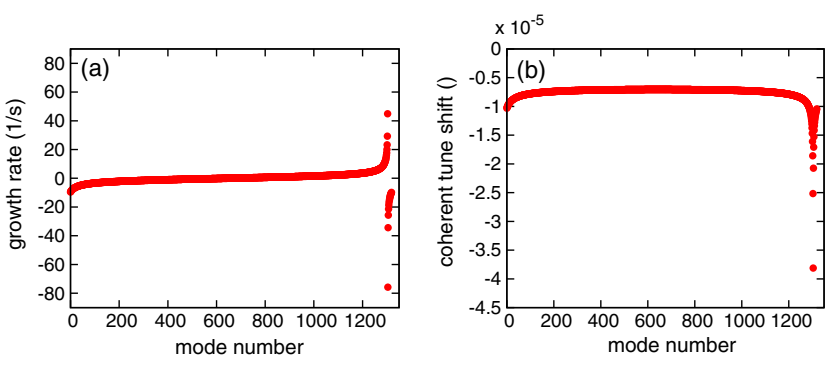

FIG. 2. Complex frequency shift for the uniform filling pattern with $M=h=1320$ at instability threshold $I_{\text {th }}^{U}=12.7 \mathrm{~mA}$ : growth rate (a); coherent tune shift (b). The fastest unstable mode $\mu=1303$ has growth rate $\tau_{\mu}^{-1}=1 / \tau_{y}$, where $\tau_{y}$ is the vertical radiation damping time.

$$
I_{\mathrm{th}}^{U}=\frac{2 T_{0} E_{0}}{\tau_{y} \max f(\mu)} .
$$

We can equivalently state that the instability threshold $I_{\text {th }}$ is obtained by equating the fastest growth time (determined by the eigenvalue with the largest imaginary part) to the radiation damping time $\tau_{y}$.

Since $\operatorname{Re} \bar{Z}_{1}^{\perp}(\omega)>0$ if $\omega>0$, and $<0$ if $\omega>0, \max f(\mu)$ can be approximated by keeping only the term with $p=-1$ and by evaluating $\operatorname{Re} \bar{Z}_{1}^{\perp}$ at its smallest, negative value, which occurs for $\mu=h-\left[\nu_{\beta}\right]-1$, where $\left[\nu_{\beta}\right]$ is the integer part of the betatron tune. With parameters given in Table II, the integer part of the vertical tune is $\left[\nu_{\beta}\right]=16$, thus the fastest growing mode is 1303 . We note that the most stable mode occurs for $\mu=h-\left[\nu_{\beta}\right]$, thus for $\mu=1304$. The corresponding complex frequency shifts read $\Omega_{1303}^{U}=$ $(-59.9,44.9) \mathrm{s}^{-1}$ and $\Omega_{1304}^{U}=(-90.7,-75.8) \mathrm{s}^{-1}$.

Equidistant filling patterns are defined by multibunch configurations in which $M$ bunches occupy symmetrically only a subset of all the available rf-buckets, i.e., where $M$ bunches are separated by the distance $d=C / M$, with $M \leq h$. The number of possible equidistant multibunch configurations, thus the possible values of $M$, is determined by the prime factorization of the harmonic number $h$. For the NSLS-II storage ring, as shown in Table II, $h=1320=$ $2^{3} \times 3 \times 5 \times 11$.

\section{A. Elementary case: $M=3$}

The complexity of the solution of the eigenvalue problem for arbitrary multibunch configurations is illustrated in the case of $M=3$ equidistant bunches, in which case the eigenvalues can be expressed in closed form. The corresponding eigenvalue problem defined by Eq. (13) reads

$$
|\mathbf{B}-\Omega \mathbf{I}|=\left|\begin{array}{ccc}
\Omega_{0}^{U}-\Omega & a \Omega_{0}^{U} & a^{*} \Omega_{0}^{U} \\
a^{*} \Omega_{1}^{U} & \Omega_{1}^{U}-\Omega & a \Omega_{1}^{U} \\
a \Omega_{2}^{U} & a^{*} \Omega_{2}^{U} & \Omega_{2}^{U}-\Omega
\end{array}\right|=0,
$$

where $a=\bar{N}_{0}-\alpha \bar{N}_{1}-\alpha^{*} \bar{N}_{2}, \quad \alpha=(1-\sqrt{3} i) / 2, \quad \bar{N}_{m}=$ $N_{m} / N_{T}$ and ${ }^{*}$ denotes complex conjugate. Eliminating $\bar{N}_{2}$ with the use of $\bar{N}_{0}+\bar{N}_{1}+\bar{N}_{2}=1$, it follows that Eq. (17) reads

$$
|\mathbf{B}-\Omega \mathbf{I}|=\Omega^{3}+b \Omega^{2}+c \Omega+d=0,
$$

with coefficients given by

$$
\begin{gathered}
b=-\operatorname{Tr} \mathbf{B}=-\left(\Omega_{0}^{U}+\Omega_{1}^{U}+\Omega_{2}^{U}\right), \\
c=-3\left(\Omega_{0}^{U} \Omega_{1}^{U}+\Omega_{0}^{U} \Omega_{2}^{U}+\Omega_{1}^{U} \Omega_{2}^{U}\right) \\
\times\left[\bar{N}_{0}\left(\bar{N}_{0}-1\right)+\bar{N}_{0} \bar{N}_{1}+\bar{N}_{1}\left(\bar{N}_{1}-1\right)\right], \\
d=27 \bar{N}_{0} \bar{N}_{1}\left(\bar{N}_{0}+\bar{N}_{1}-1\right) \Omega_{0}^{U} \Omega_{1}^{U} \Omega_{2}^{U} .
\end{gathered}
$$

The general solution of Eq. (18), which is cubic, is written in the following compact form

$$
\Omega_{\mu}=-\frac{1}{3}\left(b+\xi^{\mu} C+\frac{\Delta_{0}}{\xi^{\mu} C}\right), \quad \mu \in\{0,1,2\},
$$

where $C=\left[\left(\Delta_{1}-\sqrt{\Delta_{1}^{2}-4 \Delta_{0}^{3}}\right) / 2\right]^{1 / 3}, \Delta_{0}=b^{2}-3 c, \Delta_{1}=$ $2 b^{3}-9 b c+27 d$, and $\xi=\alpha^{*}$. With parameters listed in Table II, the complex frequency shifts $\Omega_{\mu}^{U}$ for the uniform filling pattern case with $M=3 \mathrm{read} \Omega_{0}^{U}=(-7923.2$, -8.6) $\mathrm{s}^{-1}, \Omega_{1}^{U}=(-7960.3,-70.7) \mathrm{s}^{-1}$ and $\Omega_{2}^{U}=(-7930.7$, $30.3) \mathrm{s}^{-1}$. The largest imaginary part of the eigenvalues given by Eq. (22) is shown in Fig. 3 as a function of $\bar{N}_{0}$ and $\bar{N}_{1}$. It is worth noting that the fastest instability is determined by values of $\left(\bar{N}_{0}, \bar{N}_{1}\right)$ in the neighborhood of $(1 / 3,1 / 3)$, which represents the uniform filling pattern case. Milder instabilities are identified along the isolines determined by $\bar{N}_{1}=\bar{N}_{0}$, $\bar{N}_{2}=\bar{N}_{0}$, and $\bar{N}_{2}=\bar{N}_{1}$. A slight nonuniformity in the filling pattern is therefore sufficient to improve stability.

In the case of a missing bunch, i.e., $\bar{N}_{2}=0$, using $\bar{N}_{0}+$ $\bar{N}_{1}+\bar{N}_{2}=1$ it follows from Eq. (21) that $d=0$, thus Eq. (18) reduces to

$$
\Omega\left(\Omega^{2}+b \Omega+c\right)=0
$$

with $\quad c=-3 \bar{N}_{0}\left(\bar{N}_{0}-1\right)\left(\Omega_{0}^{U} \Omega_{1}^{U}+\Omega_{0}^{U} \Omega_{2}^{U}+\Omega_{1}^{U} \Omega_{2}^{U}\right)$. It follows that one of the eigenvalues is zero, $\Omega_{2}=0$, and the other two read

$$
\begin{aligned}
\Omega_{0,1}= & \frac{\operatorname{Tr} \mathbf{B}}{2} \pm \frac{1}{2}\left[\operatorname{Tr} \mathbf{B}^{2}\right. \\
& \left.+12 \bar{N}_{0}\left(\bar{N}_{0}-1\right)\left(\Omega_{0}^{U} \Omega_{1}^{U}+\Omega_{0}^{U} \Omega_{2}^{U}+\Omega_{1}^{U} \Omega_{2}^{U}\right)\right]^{1 / 2} .
\end{aligned}
$$




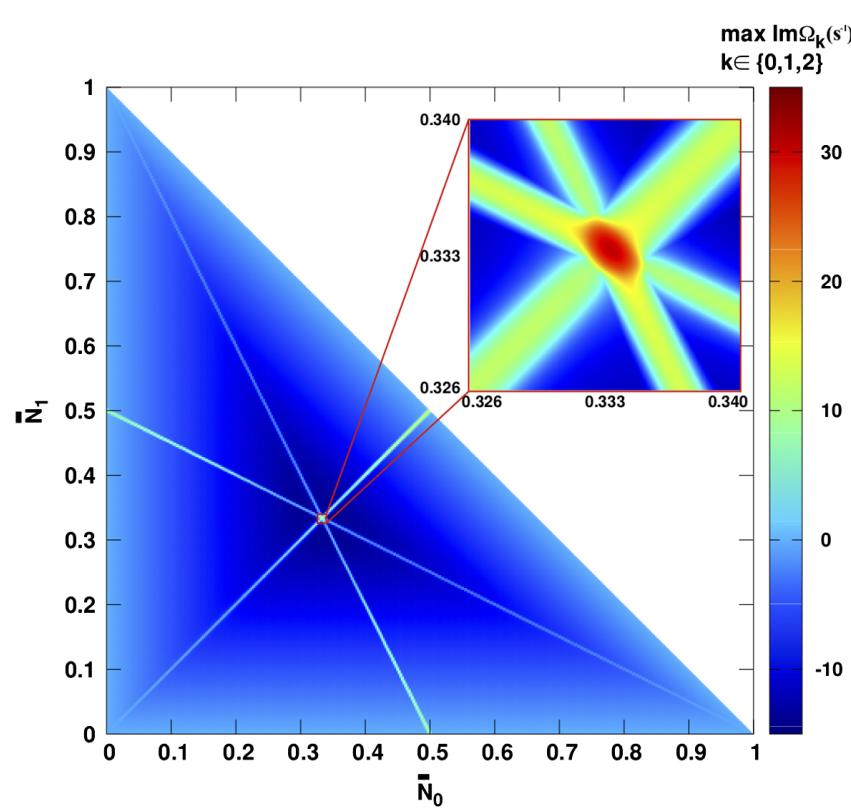

FIG. 3. Largest imaginary part (largest growth rate) of the complex frequency shifts given by Eq. (22) for the equidistant multibunch configuration with $M=3$. $I_{0}=12.7 \mathrm{~mA}$.

\section{B. Uniform filling pattern with $\boldsymbol{M} \leq \boldsymbol{h}$}

We remind that with $M$, we represent the number of bunches filling the ring equidistantly. If in addition, we assume uniformity, i.e., all bunches have the same number of particles $N_{T} / M$, we obtain uniform filling patterns with $M$ bunches. We call the configuration with $M=h$ maximal uniform filling pattern. The complex frequency shift with the largest imaginary part (largest growth rate) for all possible uniform filling patterns at $I_{0}=12.7 \mathrm{~mA}$ is shown in Fig. 4 by the red dots, with the frames (a) and (b) displaying the growth rate and coherent tune shift, respectively. For comparison, the complex frequency shift given by Eq. (32) corresponding to the uniform filling pattern case with a gap $g=M-2$, thus describing a configuration with two bunches, is also shown in Fig. 4 (blue squares). We note that uniform configurations with $M>33$ are more stable than the configuration with two bunches with a gap, thus giving a counterexample of Kohaupt argument [11], which states that the fastest instability is given by the maximal uniform filling pattern.

\section{Gap in the equidistant filling pattern:$$
\bar{N}_{\boldsymbol{m}}=\mathbf{0} \text { for } \boldsymbol{m}>\boldsymbol{M}-\boldsymbol{g}
$$

To discuss the case of a gap $g$ in the equidistant multibunch configuration with $M$ bunches, we rewrite the matrix $\mathbf{B}$ given by Eq. (13) in the form

$$
\mathbf{B}=\mathbf{\Omega}^{\mathbf{U}} \mathbf{H}, \quad H_{\mu \mu^{\prime}}=\sum_{m=0}^{M-1} \bar{N}_{m} e^{i 2 \pi m\left(\mu^{\prime}-\mu\right) / M},
$$
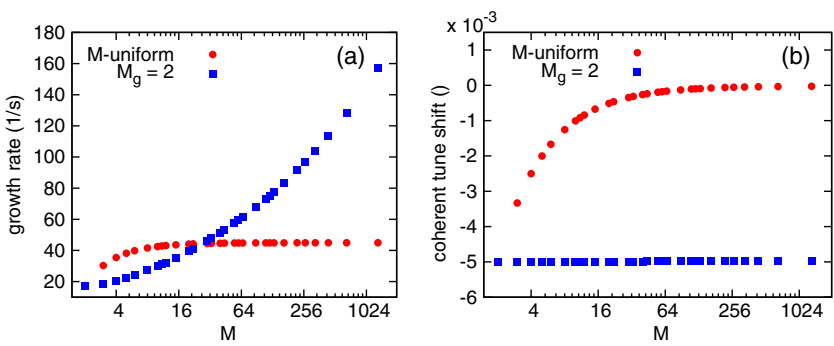

FIG. 4. Complex frequency shift with the largest imaginary part for all possible uniform filling patterns with $M \leq h$, shown by the red dots, compared with the uniform filling pattern case with a gap $g=M-2$, shown by the blue squares: growth rate (a); coherent tune shift (b). $I_{0}=12.7 \mathrm{~mA}$.

where $\boldsymbol{\Omega}^{U}=\operatorname{diag}\left(\Omega_{0}^{U}, \ldots, \Omega_{M-1}^{U}\right)$. Here we use standard results of the theory of matrices [20]. Let us assume $\boldsymbol{\Omega}^{\mathbf{U}}$ has $\operatorname{rank} M$. It follows that $\operatorname{rank}(\mathbf{B})=\operatorname{rank}(\mathbf{H})$. The Hermitian matrix $\mathbf{H}$ is circulant, therefore all the eigenvalues are real and given by

$$
\lambda_{m}=\sum_{m^{\prime}=0}^{M-1} \bar{N}_{m^{\prime}} \sum_{p=0}^{M-1} e^{i 2 \pi p\left(m^{\prime}-m\right) / M}=M \bar{N}_{m},
$$

where we made use of the orthonormality condition $\sum_{p=0}^{M-1} e^{i 2 \pi p\left(m^{\prime}-m\right) / M}=M \delta_{m^{\prime} m}$. Therefore in the case of a gap $g$ with missing bunches, i.e., $\bar{N}_{m}=0$ for $m>M-g$, $\operatorname{rank}(\mathbf{H})=M-g$, thus $\operatorname{rank}(\mathbf{B})=M-g$ as well. It follows that the characteristic polynomial of $\mathbf{B}$ reads

$$
p(\Omega)=\Omega^{g} \sum_{k=0}^{M-g} c_{k} \Omega^{k} .
$$

We have therefore proved that the number of zero-eigenvalues is equal to the length of the gap $g$.

With the use of the Fadded-LeVerrier algorithm [20], we construct now an explicit form of $p(\Omega)$ for the case $g=M-2$, namely

$$
p(\Omega)=\Omega^{M-2}\left(c_{M} \Omega^{2}+c_{M-1} \Omega+c_{M-2}\right),
$$

where $c_{M}=1, \quad c_{M-1}=-\operatorname{Tr} \mathbf{B}, \quad$ and $\quad c_{M-2}=-\left(\operatorname{Tr} \mathbf{B}^{2}-\right.$ $\left.(\operatorname{Tr} \mathbf{B})^{2}\right) / 2$. Using the identities

$$
(\operatorname{Tr} \mathbf{B})^{2}=\sum_{\mu=0}^{M-1} \Omega_{\mu}^{U 2}+2 \sum_{\mu=0}^{M-1} \sum_{\mu^{\prime}=\mu+1}^{M-1} \Omega_{\mu}^{U} \Omega_{\mu^{\prime}}^{U},
$$

$$
\begin{aligned}
\operatorname{Tr} \mathbf{B}^{2} & =\sum_{\mu=0}^{M-1} \sum_{\mu^{\prime}=0}^{M-1} \Omega_{\mu}^{U} \Omega_{\mu^{\prime}}^{U}\left|H_{\mu, \mu^{\prime}}\right|^{2} \\
& =\sum_{\mu=0}^{M-1} \Omega_{\mu}^{U 2}+2 \sum_{\mu=0}^{M-1} \sum_{\mu^{\prime}=\mu+1}^{M-1} \Omega_{\mu}^{U} \Omega_{\mu^{\prime}}^{U}\left|H_{\mu, \mu^{\prime}}\right|^{2},
\end{aligned}
$$


it follows that

$$
\begin{aligned}
\operatorname{Tr} \mathbf{B}^{2} & =(\operatorname{Tr} \mathbf{B})^{2}+2 \sum_{\mu=0}^{M-1} \sum_{\mu^{\prime}=\mu+1}^{M-1} \Omega_{\mu}^{U} \Omega_{\mu^{\prime}}^{U}\left(\left|H_{\mu, \mu^{\prime}}\right|^{2}-1\right) \\
& =(\operatorname{Tr} \mathbf{B})^{2}\left(1+\frac{1}{2} \sum_{\mu=0}^{M-1} \sum_{\mu^{\prime}=\mu+1}^{M-1} g_{\mu \mu^{\prime}} \Omega_{\mu}^{U} \Omega_{\mu^{\prime}}^{U}\right),
\end{aligned}
$$

where $g_{\mu \mu^{\prime}}=4\left(\left|H_{\mu \mu^{\prime}}\right|^{2}-1\right) /\left((\operatorname{Tr} \mathbf{B})^{2}\right)$, thus the eigenvalues of Eq. (28) read $\Omega_{k}=0$ for $k \geq 2$ and

$$
\Omega_{0,1}=\frac{\operatorname{Tr} \mathbf{B}}{2}\left(1 \pm \sqrt{1+\sum_{\mu=0}^{M-1} \sum_{\mu^{\prime}=\mu+1}^{M-1} g_{\mu \mu^{\prime}} \Omega_{\mu}^{U} \Omega_{\mu^{\prime}}^{U}}\right)
$$

In the case $\bar{N}_{0}=\bar{N}_{1}=1 / 2, \bar{N}_{m}=0$ for $m \geq 2$, we have $g_{\mu \mu^{\prime}}=\left(\cos 2 \pi\left(\mu-\mu^{\prime}\right) / M-1\right) / 2$. For the case $M=3$, by noting that $c_{01}=c_{02}=c_{12}=-3$, it follows that Eq. (32) reduces to Eq. (24) with $\bar{N}_{0}=0.5$. With parameters listed in Table II, the eigenvalues read $\Omega_{0}=(-11892.7,18.7) \mathrm{s}^{-1}$, $\Omega_{1}=(-11921.7,-67.7) \mathrm{s}^{-1}$, and $\Omega_{2}=0$. The fastest instability has therefore a growth rate $\operatorname{Im} \Omega_{0}=18.7 \mathrm{~s}^{-1}$. For comparison, the growth rate of the fastest growing mode of the uniform filling pattern case given by Eq. (22) is bigger, i.e., $\operatorname{Im} \Omega_{2}^{U}=30.4 \mathrm{~s}^{-1}$. The eigenvalue with the largest imaginary part for all possible uniform filling patterns with $M \leq h$ and $I_{0}=12.7 \mathrm{~mA}$ is shown by the blue squares in Fig. 4(a), with the coherent tune shift shown in Fig. 4(b).

\section{MEASUREMENTS WITH A GAP IN THE MAXIMAL UNIFORM FILLING PATTERN}

The theory of the coupled-bunch instability for arbitrary multibunch configurations has been benchmarked against measurements at the NSLS-II storage ring. In benchmarking theory with measurements, the theoretical instability threshold is obtained by equating the fastest growth time (determined by the eigenvalue with the largest imaginary part) to the radiation damping time $\tau_{y}$. This is equivalent to assume that Eq. (15) has an extra multiplicative factor $\exp \left(-t / \tau_{y}\right)$. This assumption has been verified in the benchmark of Eq. (13) against Vlasov-Fokker-Planck simulations with space [16], where a detailed discussion of the procedure adopted to calculate numerically the growth rates can be found [21].

The theoretical instability threshold for an arbitrary multibunch configuration, $I_{\text {th }}$, is determined by calculating first the theoretical instability threshold for the uniform filling case, $I_{\mathrm{th}}^{U}$, which is defined by Eq. (16). With $\mu=$ 1303 and $\tau_{y}=22.5 \mathrm{~ms}$, it follows $I_{\text {th }}^{U}=12.7 \mathrm{~mA}$ (see Fig. 2). Second, to determine $I_{\text {th }}$, we solve the eigenvalue equation (13) replacing $I_{0}$ with $I_{\text {th }}^{U}$ in Eq. (14). The corresponding matrix $\mathbf{B}$, with an abuse of notation, is denoted $\mathbf{B}\left(I_{\mathrm{th}}^{U}\right)$. Since the instability threshold $I_{\mathrm{th}}$ is determined by solving Eq. (13) with $\mathbf{B}\left(I_{\mathrm{th}}\right)$, using the fact that $\mathbf{B}\left(I_{\mathrm{th}}\right) / \mathbf{B}\left(I_{\mathrm{th}}^{U}\right)=I_{\mathrm{th}} / I_{\mathrm{th}}^{U}$, it follows that the eigenvalues of $\mathbf{B}\left(I_{\mathrm{th}}\right)$ are $I_{\mathrm{th}} / I_{\mathrm{th}}^{U}$ times the eigenvalues of $\mathbf{B}\left(I_{\mathrm{th}}^{U}\right)$, thus

$$
I_{\mathrm{th}}=I_{\mathrm{th}}^{U} \frac{\tau_{g}}{\tau_{y}},
$$

where $\tau_{g}$ is the growth time of the fastest growing mode, determined by the eigenvalue of $\mathbf{B}\left(I_{\mathrm{th}}^{U}\right)$ with the largest imaginary part. The measurements have been performed with a gap in the maximal uniform filling pattern, with the instability driven by the vertical resistive wall impedance with parameters listed in Table I. The storage ring has been configured with the 3DWs lattice at zero linear chromaticity [22], with the gaps of the three damping wigglers closed and all the other insertion device gaps open. The coupled-bunch instability threshold as a function of the gap has been determined experimentally from the stability of Beam Position Monitor (BPM) measurements as discussed in Fig. 5 where the measurements performed at different average currents are shown for the case $M_{g}=M-g=$ 600 (left frames) and $M_{g}=M-g=1200$ (right frames), where $g$ is the gap in the maximal $(M=1320)$ uniform filling pattern, with the measurements of all the other cases showing a similar behavior. The frames (a)-(h) show the time evolution of the average bunch centroid position over $50 \mathrm{~K}$ turns, as detected from 6 of the 180 regular BPMs distributed around the NSLS-II storage ring, with the frames (a)-(d) and (e)-(h) showing unstable and stable betatron motion, respectively, characterized by a peak-topeak variation above $10 \mu \mathrm{m}$ in the unstable case, and a peak-to-peak variation well below $5 \mu \mathrm{m}$, at the noise level, in the stable case. The transition from stable to unstable motion can be seen more clearly in frequency domain, as shown in the bottom frames (i) and (1), where the average spectra of the time domain BPM measurements are calculated with a Discrete Time Fourier Transform (DTFT) [23]. For a good control of the spectral leakage, a window function of length $L=40 \mathrm{k}$ turns has been used, and the DTFT has been computed with a sampling rate $\Delta f_{y}=\Delta \nu_{y} f_{0}=3.79 \mathrm{~Hz}$, where $f_{0}$ is the revolution frequency. In frequency domain, the peak in the spectra shown above instability threshold exceed 100 times the noise level shown by the spectra below threshold. The peak in the spectrum close to the vertical tune shown by the blue trace gives an upper bound to the instability threshold. We define the measured instability threshold $I_{\text {th }}$, as plotted in Fig. 6(a), as the arithmetic mean between the adjacent stable and unstable currents as shown in (i) and (1) by the green and blue traces, respectively. Accordingly, for the two cases shown in Fig. 5, we have the following threshold currents: $I_{\text {th }}=10.5 \mathrm{~mA}$ for $M_{g}=600$ ((i)) and $I_{\text {th }}=$ $12.6 \mathrm{~mA}$ for $M_{g}=1200((1))$. All the measurements have 

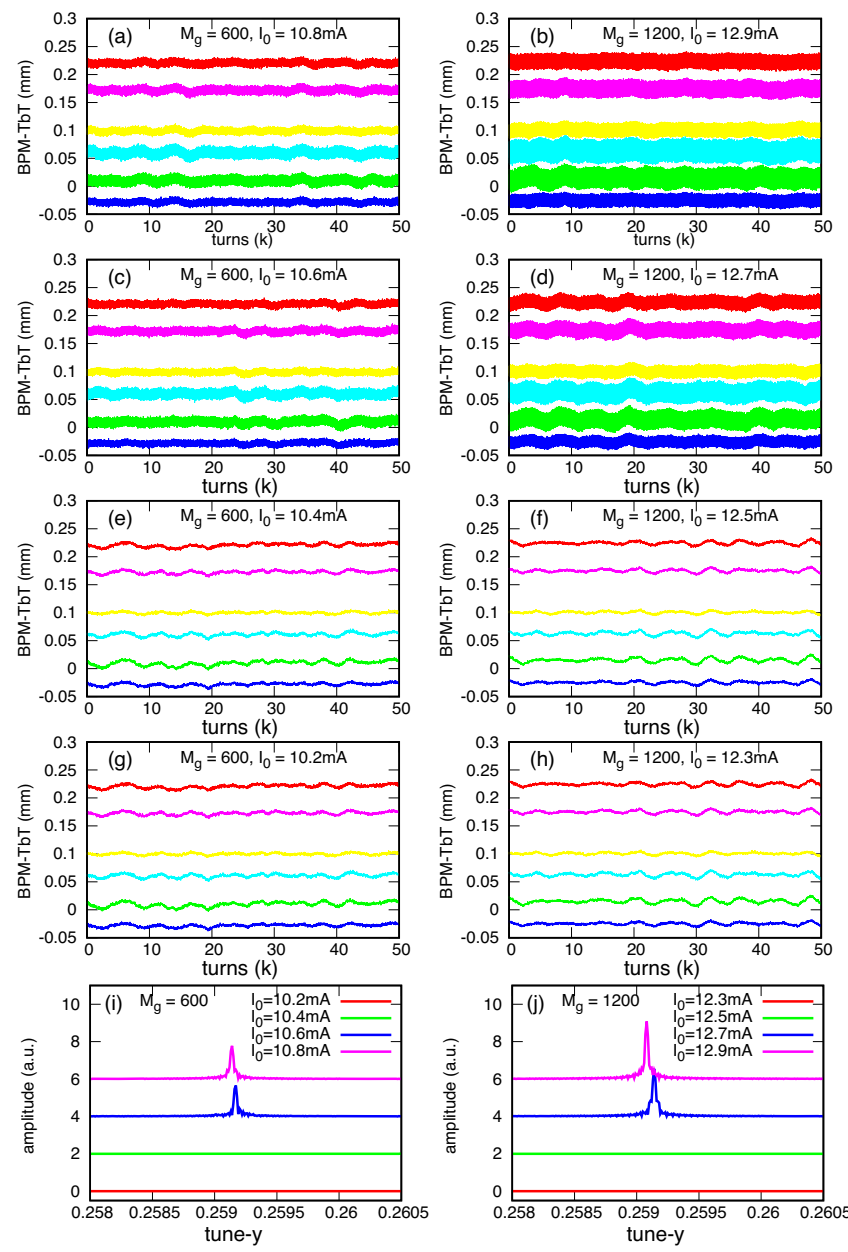

FIG. 5. Measurements at different average currents for the case $M_{g}=M-g=600$ (left frames) and $M_{g}=M-g=1200$ (right frames), where $g$ is the gap in the maximal $(M=1320)$ uniform filling pattern. (a)-(h) time evolution of the average bunch centroid position over $50 \mathrm{~K}$ turns, as detected from 6 of the 180 regular BPMs distributed around the NSLS-II storage ring. (a)-(d) and (e)-(h) showing unstable and stable betatron motion respectively. (i) and (j): average spectra of the time domain BPM measurements showing the transition from stable to unstable motion.

been performed with the same step change in the average current $\Delta I_{0}=0.2 \mathrm{~mA}$. Some of the multibunch configurations, as measured from the NSLS-II filling pattern monitor, are shown in Fig. 6(b). The measured instability thresholds as a function of $M_{g}=M-g$ are plotted in Fig. 6(a), where they are shown to be in close agreement with the numerical solution of the eigenvalue problem given by Eq. (13). It is worth noting that the instability threshold decreases with the increase of the gap $g$, with the most stable configuration given by the maximal uniform filling pattern. The close agreement between theory and simulations, besides benchmarking the eigenanalysis for arbitrary multibunch configurations, validates the accuracy of the impedance model used.
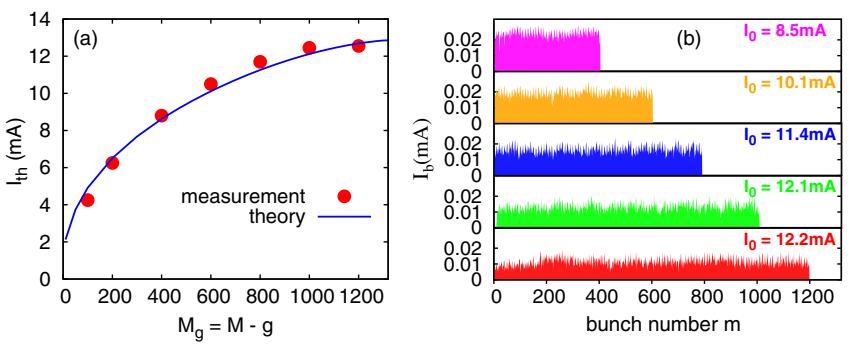

FIG. 6. (a) Vertical coupled-bunch instability threshold $I_{\text {th }}$ as a function of $M_{g}=M-g$, where $g$ is the gap in the maximal ( $M=1320)$ uniform filling pattern: comparison between theory and measurements. The blue trace shows the analytical result obtained by equating the fastest growth time (determined by the eigenvalue with the largest imaginary part) to the radiation damping time $\tau_{y}=22.5 \mathrm{~ms}$; the red dots represent the measured thresholds, obtained as the arithmetic mean of the currents slightly below and above threshold, as shown in Fig. 5. (b) Bunch trains close to instability threshold as measured from the NSLS-II filling pattern monitor: single bunch current $I_{b}=I_{0} / M_{g}$ as a function bunch number $m$.

\section{CONCLUSIONS}

We discussed theoretically the coupled-bunch instability for arbitrary multibunch configurations and benchmarked its predictions against measurements performed at the NSLS-II storage rings. Besides helpful for the design of hybrid modes of operations in storage rings, the results discussed in this paper, in particular the closed formulas presented for some special cases, might be useful for benchmarking beam dynamics codes that model the coupled-bunch instability for arbitrary filling patterns. The primary goal of the paper has been to present a clear comparison with measurements of the coupled-bunch instability theory, setting the experimental conditions in order to isolate the coupled-bunch interaction has the dominant effect driving the instability. To this end, we set the chromaticity to zero in order to avoid the chromatic head-tail damping driven by short-range wakefields, that would have required a broadband impedance model thus complicating the analysis. For the same reason, we did not include a feedback model. A partial analysis of such effects has been done in Ref. [24], with single-bunch studies of the combined effect of chromaticity and feedback on the transverse head-tail instability, and in Ref. [25], with a numerical study of the stabilizing effect of the chromatic head-tail damping on the transverse coupled-bunch instability. The extension of the theoretical analysis discussed in this paper to include the aforementioned effects, as well as further benchmarking against measurements and particle tracking codes, will be the subject of future work.

\section{ACKNOWLEDGMENTS}

We gratefully acknowledge support by DOE under Contract No. DE-SC0012704. 
[1] R. Nagaoka and K. L. Bane, Collective effects in a diffraction-limited storage ring, J. Synchrotron Radiat. 21, 937 (2014).

[2] Brookhaven National Laboratory, NSLS-II CDR, 2006, https://www.bnl.gov/nsls2/project/CDR/Cover.pdf.

[3] The two terms multi-bunch configuration and filling pattern are used synonymously throughout the paper.

[4] T. Olsson, Timing modes for the MAX IV storage rings, Ph.D. thesis, Lund University, 2018, https://lucris.lub.lu.se/ ws/portalfiles/portal/42857908/teresia_phd_thesis_kappa .pdf.

[5] J. Xie et al., First experimental demonstration of timeresolved X-ray measurements with next-generation fasttiming MCP-PMT, Nucl. Instrum. Methods Phys. Res., Sect. A 927, 287 (2019).

[6] R. Müller et al., BESSY II supports an extensive suite of timing experiments, in Proceedings of the 2016 International Particle Accelerator Conference, Busan, Korea (2016), https://accelconf.web.cern.ch/ipac2016/ papers/wepow011.pdf.

[7] M. Cammarata et al., Chopper system for time resolved experiments with synchrotron radiation, Rev. Sci. Instrum. 80, 015101 (2009).

[8] M. G. Silly et al., Pump-probe experiments at the TEMPO beamline using the low-a operation mode of Synchrotron SOLEIL, J. Synchrotron Radiat. 24, 886 (2017).

[9] A. W. Chao, Physics of Collective Beam Instabilities in High Energy Accelerators, 1st ed. (Wiley-VCH, New York, 1993).

[10] K. Y. Ng, Physics of Intensity Dependent Beam Instabilities (World Scientific Publishing Company, Singapore, 2005).

[11] R. D. Kohaupt, On multi-bunch instabilities for fractionally filled rings, Report No. DESY-85-139, DESY, Hamburg, Germany, 1985, https://lib-extopc.kek.jp/preprints/PDF/ 1986/8603/8603031.pdf.

[12] J. S. Berg, Coherent modes for multiple non-rigid bunches in a storage ring, Ph.D. thesis, Stanford University, SLAC-R-478 (1996), https://www.slac.stanford.edu/pubs/ slacreports/reports06/slac-r-478.pdf.

[13] S. Prabhakar, New diagnostic and cures for coupled-bunch instabilities, Report No. SLAC-R-554, 2000, https://www .slac.stanford.edu/pubs/slacreports/reports03/slac-r-554 .pdf.

[14] S. Prabhakar, J. D. Fox, and D. Teytelman, Curing Coupled-Bunch Instabilities with Uneven Fills, Phys. Rev. Lett. 86, 2022 (2001).

[15] A. Burov, Nested head-tail Vlasov solver, Phys. Rev. ST Accel. Beams 17, 021007 (2014).

[16] G. Bassi, A. Blednykh, and V. Smaluk, Self-consistent simulations and analysis of the coupled-bunch instability for arbitrary multibunch configurations, Phys. Rev. Accel. Beams 19, 024401 (2016).

[17] The thickness of the storage ring components contributing to the coupled-bunch instability threshold is much smaller than the skin depth evaluated at $\omega_{q}=(1-q) \omega_{0}$, allowing the penetration of the electromagnetic fields at frequencies higher then $\omega_{q}$.

[18] A. W. Chao et al., Handbook of Accelerator Physics and Engineering, 2nd ed. (World Scientific, Singapore, 2013).

[19] The coupling factor $A_{x}=e c /\left(T_{0} E_{)}\right)$, as given in Ref. [16], is valid for a storage ring with constant beta function $\beta_{\perp}$, which is related to the betatron frequency $\omega_{\beta}$ by the equation $\omega_{\beta} / c=1 / \beta_{\perp}$. Since the total average impedance $\bar{Z}_{1}{ }^{\perp}$, and thus the total average wakefield $\bar{W}_{1}$, is constructed by weighting the individual impedance components by the average of the beta function over the length of the impedance structure, as discussed in Sec. II, we rescale $A_{x}$ by the factor $\omega_{\beta} / c$, thus $A_{x}=e \omega_{\beta} /\left(T_{0} E_{0}\right)$.

[20] F. R. Gantmacher, The Theory of Matrices (Chelsea Publishing, NY, 1960).

[21] The numerical growth rates have been found by fitting with an exponential the envelope of the betatron oscillations of the bunch centroids. From the definition of the multi-bunch mode $x_{m}^{(\mu)}(t)$ given in Eq. (10), it follows that an arbitrary multi-bunch configuration can be expressed as sum of multi-bunch modes $x_{m}(t)=\frac{1}{2} \sum_{\mu=0}^{M-1} x_{m}^{(\mu)}(t)$. In the uniform filling pattern case, the modes $\tilde{x}_{\mu}$ are uncoupled, thus the growth rate of mode $\tilde{x}_{\mu}$ is calculated via configuring the initial state in the multi-bunch $x_{m}^{(\mu)}(0)$. In the arbitrary filling pattern case, the modes $\tilde{x}_{\mu}$ are coupled and their expression is given by Eq. (15). All modes are affected by the eigenvalue with the largest imaginary part, with the degree of coupling given by the initial conditions determined by the eigenvectors.

[22] The linear chromaticity has been set to zero to avoid the slow-had tail damping induced by short-range wakefields, which have been observed in studies to be strong enough to affect the decoherence process of bunches with single bunch current as low as few $\mu A$.

[23] A. V. Oppenheim, R. W. Schafer, and J. R. Buck, DiscreteTime Signal Processing (Prentice-Hall, NJ, 1999).

[24] V. Smaluk, G. Bassi, A. Blednykh, and A. Khan, Combined effect of chromaticity and feedback on transverse head-tail instability, Phys. Rev. Accel. Beams 24, 054401 (2021).

[25] G. Bassi, A. Blednykh, W. Cheng, F. Gao, J. Rose, and D. Teytelman, Analysis of coupled-bunch instabilities for the NSLS-II storage ring with $500 \mathrm{MHz}$ 7-cell PETRA-III cavity, Nucl. Instrum. Methods Phys. Res., Sect. A 810, 151 (2016). 